American Journal of Pharmaceutical Education 2016; 80 (8) Article 129.

\title{
STATEMENT
}

\section{The Role of Pharmacists and Pharmacy Education in Point-of-Care Testing}

\author{
James P. Kehrer, $\mathrm{PhD},{ }^{\mathrm{a}}$ Deborah E. James, $\mathrm{PhD}^{\mathrm{b}}$ \\ ${ }^{a}$ University of Alberta Faculty of Pharmacy and Pharmaceutical Sciences, Edmonton, Alberta, Canada \\ ${ }^{\mathrm{b}}$ University of Alberta Faculty of Medicine and Dentistry, Edmonton, Alberta, Canada \\ Submitted November 20, 2015; accepted February 3, 2016; published October 25, 2016.
}

\begin{abstract}
Point-of-care testing (POCT) is defined as laboratory testing conducted close to the site of patient care. Although performed originally primarily by clinical staff for acute conditions, recent advances in technology have made such testing possible for disease screening and prevention across a wide range of conditions in virtually any setting, and often by individuals with little or no training. With the ongoing evolution in POCT, numerous concerns have arisen about the quality and accuracy of the tests, comparability between multiple tests for the same endpoint, interpretation of test results, and whether and how results should be used for therapeutic decisions and included in a patient's medical record. The pharmacist is well-positioned to manage and interpret POCT performed outside of the usual clinical settings. However, educational and regulatory changes are needed to enable pharmacists to take on this emerging activity effectively.
\end{abstract}

Keywords: point-of-care testing, clinical test, medical device, laboratory test, pharmacy education

\section{INTRODUCTION}

Point-of-care testing (POCT) is defined as laboratory testing conducted close to the site of patient care. While various POCT kits have been used for years (eg, blood glucose, urine ketones, pregnancy), research and discussions about POCT focus on the use of new technologies that can test for blood coagulation times, blood gas composition, cardiac enzyme profiles, standard electrolytes, and the presence of infectious entities including influenza, human immunodeficiency virus (HIV), hepatitis $\mathrm{C}$, and group A streptococcus. These tests are increasingly being administered by trained staff in hospitals, clinics, and other professional health-care settings, including pharmacies. ${ }^{1}$ Rapid advances in testing technology, including the development of easy-to-use portable healthmonitoring devices, means that such testing can now be done by individuals with little or no training and in virtually any setting, including a patient's home. This manuscript focuses on the role of pharmacists and pharmacy education in POCT outside of traditional clinical settings.

With the ongoing evolution in POCT, numerous concerns have arisen about the quality and accuracy of the tests, comparability between multiple tests for the same endpoint (ie, each test may be internally consistent but provide different absolute values, making longitudinal

Corresponding Author: James P. Kehrer, Pharmacy and Pharmaceutical Sciences, MS 2-35F, University of Alberta, 8613 - 114th St., Edmonton, AB T6G 2H1. Tel: 780-4920204. E-mail: jkehrer@ualberta.ca data more difficult to evaluate if the same manufacturer is not used each time), interpretation of the results, how to use the results for clinical decision-making, and whether and how to include the results in a patient's medical record. Although performing a point-of-care test may be done in many settings, optimal use of the generated data requires appropriate interpretation of the results and communication among the multidisciplinary health-care team and with the patient. Communication among these stakeholders with different levels of understanding of human physiology, biochemistry, disease, and test characteristics offers multiple opportunities for misunderstanding, a concern that is compounded when patients are self-testing. In terms of education, it is important that pharmacists become knowledgeable about the mechanics and data of POCT and are able to communicate this information to patients and other health-care providers.

The potential impact of POCT on pharmacy practice and patient care in ambulatory settings was highlighted in August 2015 at an inaugural conference that brought together POCT manufacturers and pharmacy professionals. ${ }^{2}$ Although POCT performed in clinical settings appears to have the potential to positively impact patient outcomes by providing rapid results and potentially earlier diagnosis and subsequent treatment (including lifestyle modifications to avoid pharmacotherapy), data supporting such uses remain limited and inconclusive. ${ }^{3}$ This article describes some of the issues surrounding POCT, particularly POCT performed outside traditional health care settings, and makes the case for pharmacists to 


\section{American Journal of Pharmaceutical Education 2016; 80 (8) Article 129.}

embrace this technology as a means to enhance patient care. At the same time, if pharmacists are to embrace POCT effectively, pharmacy education must evolve to include more knowledge about this type of testing.

\section{EDUCATIONAL STANDARDS}

In Canada, Standard 27 of the 2013 Canadian Council for Accreditation of Pharmacy Programs (CCAPP) accreditation standards states, "The core curriculum must include a balance of coursework in biomedical sciences, pharmaceutical sciences, behavioral, social and administrative sciences, and clinical sciences and practice skills." Criterion 27.5 under this standard states, "Clinical practice skills must be developed through appropriate environments such as practice, laboratory or simulation experiences. This should include but is not limited to collaborative care with other health-care providers, compounding, diagnostic and point-of-care testing...."

In the United States, accreditation standards and key elements of the 2016 Standards from the Accreditation Council for Pharmacy Education (ACPE) do not specifically mention POCT. However, required elements of the didactic curriculum include the "application of clinical laboratory data to disease state management, including screening, diagnosis, progression, and treatment evaluation." Thus, both Canadian and USA accrediting bodies recognize the importance of educating student pharmacists about laboratory data and its application to disease monitoring. What appears to be lacking within pharmacy schools is a focused effort to enhance this educational need in the face of burgeoning POCT in the pharmacy environment.

\section{POCT KITS AND DEVICES}

In the early days of western medicine, health care was delivered primarily in the patient's home and what limited testing was available was provided directly at this local point of care. As the science and technology of medicine developed rapidly in the mid- to late twentieth century, care shifted to hospitals with an emphasis on treating injuries and curing acute disease. At this time, centralized laboratories were established for the analysis of numerous patient samples using standardized techniques and complex equipment. As technology improved, automation was added, further standardizing the results.

Today, ongoing research is identifying an increasing number of disease biomarkers, and additional tests for these biomarkers are continually being developed. Furthermore, there is an increased emphasis on wellness and preventive activities as evidenced by the rapid expansion of fitness trackers, which are effectively point-of-care tests, and some of these have the ability to measure rather sophisticated clinical endpoints. This potential is being exploited by the development of smart phone apps designed to collect data to promote healthier behaviors. Despite the extensive marketing and use of such devises, research continues to be inconclusive regarding the health and economic value of such advancements. ${ }^{4}$

Technological drivers of POCT for consumer medicine include advances in assay automation, low cost sensors, and instrument miniaturization, as well as access to cloud computing. However, while many routine laboratory tests provide critical information about a patient's health status, tests are sometimes ordered just because they are available, and new tests are sometimes implemented without adequate justification for their prognostic or economic value. Nevertheless, as the technology advances, opportunities are being seized by manufacturers to make POCT available directly to consumers, a scenario that will undoubtedly affect pharmacists through patient purchases, questions, and requests for clinical services.

Point-of-care devices have been used for several decades, but until recently on a limited basis in the hospital or other acute care settings when rapid analysis is sometimes needed (eg, in intensive care units for methicillinresistant Staphylococcus aureus, procalcitonin, and rapid diagnostic testing for bacteria) and for simple home testing such as diabetes and pregnancy. In the intensive care environment, POCT can improve patient care. For example, serial analyses of arterial blood lactate with a handheld analyzer, coupled with a specific management protocol, resulted in a marked reduction in mortality for infants and neonates undergoing heart surgery. ${ }^{5}$ It also has value in diagnosing and treating HIV infection in resource-constrained countries. ${ }^{6}$ Recently, the potential advantages of POCT for international normalized ratio (INR) monitoring led to the development of guidelines in Canada for its use in patients on oral anticoagulant therapy. ${ }^{7}$

In a review examining the impact of POCT, St. John concluded that POCT alone is often insufficient to achieve health benefits and that changes in care processes are also required. ${ }^{8}$ However, the advances in POCT over the past six years indicate a need for additional research to address the clinical and economic impact of new technologies. Howick et al's survey indicated that primary care physicians in Australia, Belgium, The Netherlands, the United Kingdom, and the United States wanted POCT to help them diagnose a range of acute (infections, cardiac disease, pulmonary embolism/deep vein thrombosis) and chronic (diabetes, anemia) conditions. ${ }^{9}$ Of note, the POCT kits used most frequently in the primary care setting (blood glucose, urine pregnancy, and urine leucocytes/nitrites) 


\section{American Journal of Pharmaceutical Education 2016; 80 (8) Article 129.}

only partially correspond with the conditions for which these physicians would like immediate diagnoses. Although there were differences across countries, there appeared to be a need for POCT kits to assist clinicians with immediate decisions regarding urgent referrals or treatment. What is not yet clear is whether the range of POCT kits becoming available - from infectious disease to cholesterol and INR tests - should be performed by health care professionals in various settings and what demand individuals will have for self-testing and monitoring of acute or chronic conditions.

An increasing number of POCT kits and devices are available for general use (Appendix 1). These allow patient diagnoses in a pharmacy (eg, streptococcal and influenza tests), a physician's office, an ambulance, at home, or a hospital. The tests may give results directly or require sending a sample to a central laboratory for analysis with results returned to the patient or health-care provider, often within 24 hours. Assuming the results are accurate and interpreted appropriately, care can then occur in a timely manner.

\section{REGULATORY ISSUES}

Point-of-care testing kits and equipment are considered "medical devices" and must be evaluated and approved by government agencies in most countries before the results can be considered for clinical decisionmaking. In the United States, commercial clinical laboratory tests are regulated by the Food and Drug Administration (FDA) to ensure they meet safety and effectiveness standards. A product is considered safe when its clinical benefit to the patient is judged to exceed the potential risk associated with using the technology, and effectiveness is established when clinically significant results can be obtained. Medical devices are categorized into classes based on the degree of risk posed to the patient: class I devices pose low risk, class II devices pose moderate risk, and class III devices pose the greatest risk. In Canada, a similar process exists and involves obtaining a Health Canada medical device license as governed by Canada's Food and Drug Act. Other countries may accept the FDA decision, have comparable agencies responsible for approving the use of clinical laboratory tests, or simply allow the use of such kits without approval.

In the United States, the Centers for Medicare \& Medicaid Services (CMS) regulate all laboratory testing performed on humans according to the Clinical Laboratory Improvement Amendments (CLIA) passed by Congress in 1988. There are three regulatory categories under CLIA, based on the potential risk to public health associated with the test of interest. Tests can fall into the waived category, or into a moderate or high complexity category.
For a test to be considered CLIA-waived, it must employ sufficiently simple and accurate methodologies so the risk of erroneous results is negligible, or there is no significant risk of harm to the patient if the test is performed incorrectly. There are more than 120 waived tests, ${ }^{10}$ and these are the types of tests making their way into pharmacy practice because of their minimal regulatory oversight.

To perform CLIA-waived tests in the United States, a pharmacy must apply for and maintain a CLIA Certificate of Waiver and CLIA license. This involves filling out the appropriate form, sending the application to the State Survey Agency for CMS, paying biennial fees, following manufacturer test guidelines for test procedures and quality control, and agreeing to inspections by CMS. ${ }^{11}$ The amendments state that waived laboratories must follow "good laboratory practice" when performing tests, which address issues of proper physical environment and recording of test results and patient information in a retrievable file. ${ }^{12}$ Further considerations for pharmacists wishing to perform POCT in pharmacies are standards set by the US Occupational Safety and Health Administration (OSHA) as well as any individual state standards that may exist aimed at ensuring a safe and healthy work environment for employers. These standards outline protocols necessary to minimize the risk of employee exposure to blood and infectious materials and include the use of personal protective equipment (eg, gloves, laboratory coats), work practices to prevent needle-sticks, and provision of hepatitis $B$ vaccination for employees. Other important requirements relate to employee training, recordkeeping, and the existence of an exposure control plan in the event of exposure. ${ }^{12}$

The number of tests receiving a waiver will increase as technology improves and more "noncritical" endpoints are identified. An example is the genetic test for Bloom Syndrome that received FDA approval as a "direct-toconsumer genetic test" in early 2015. Although the disease is rare, the approval from the FDA is an indicator of how this agency may proceed with regulating the many new genetic tests arising from the revolution in DNA sequencing technology.

A similar revolution is underway in biochemical and molecular testing where the technology enables simultaneous assessment of a wide range of diseases. These labon-a-chip devices may include one or many laboratory functions on a single chip of only millimeters to a few square centimeters in size, and use extremely small fluid volumes. Although not yet available for personal use, labon-a-chip manufacturers promise results in minutes and that the technology could be used to test for numerous conditions simultaneously including diabetes, blood disorders, HIV, liver and kidney function, sexually transmitted 


\section{American Journal of Pharmaceutical Education 2016; 80 (8) Article 129.}

diseases, coagulation disorders, and more. At the same time, the claims made for such tests are not always realistic and must be examined rigorously, including peer-reviewed research. ${ }^{13}$

\section{ACCESS TO LABORATORY TESTS}

All too often, health care is impacted adversely because the patient finds the care difficult to access, unaffordable, or is reluctant to seek help for other reasons. This access issue is also endemic in many third world countries for economic and logistical reasons. POCT has the potential to empower a broader range of clinicians, or even the patients themselves, to obtain clinical data, and thus has the potential to improve diagnoses and health-care delivery, and to address the challenges of accessibility and health disparities. It is noteworthy that a national POCT program in Australia relating to diabetes management in an aboriginal population demonstrated that it provided a convenient and accessible service that empowered Aboriginal Health Workers to have a direct role in the care of their diabetes patients. ${ }^{14}$

In addition to the value of POCT in acute care settings, a desired outcome of using POCT kits in nontraditional settings is a shift from treating acute disorders to early detection of disease and prevention. POCT also has the potential to encourage more effective management of multiple chronic conditions by providing information on a patient's genomics for use in tailoring therapy. Point-ofcare testing may enable more frequent access to critical data in easily accessible and less expensive medical settings, or even the patient's home. However, success depends on the development of accurate, inexpensive devices that are simple to operate. In addition, success will require regulatory changes, acceptance by patients, and a focused effort among clinicians, such as pharmacists, to embrace this opportunity in a collaborative fashion.

\section{ROLE OF PHARMACISTS}

As new tests and technologies emerge, POCT provides an excellent opportunity for pharmacists to adopt a greater role in patient care; one that builds upon their knowledge and expertise in drugs and drug therapy, and their access to patients. In fact, pharmacists have been providing POCT, such as those for influenza, streptococcal infections, glycosylated hemoglobin (A1c) and cholesterol screenings, for years. An emerging area of clinical data where pharmacists will have an explicit and important role is pharmacogenomics. Although not yet mainstream, POCT devices that measure a person's drug metabolizing abilities are emerging, and pharmacists, as medication experts, should be at the forefront of this testing landscape. This area also raises interesting questions around the potential need for pharmacists to obtain patient consent for genomic testing.

One model being promoted for engaging pharmacists more broadly in POCT is "permissionless innovation in practice." 15 This model asserts that experimentation with new technologies such as POCT should generally not require regulatory permission. While intriguing, patient safety is paramount and a total absence of regulation seems unlikely. In Alberta, Canada, because any licensed pharmacist listed in the Provincial Provider Registry has the authority to order laboratory tests, most pharmacists can engage in POCT. Other jurisdictions may need to advocate for additional collaborative practice authority or other regulatory changes to enable optimal use of POCT by pharmacists. However, as patients themselves become involved in POCT through approved (or perhaps unapproved) kits, this distinction may become blurred.

Weidle et al demonstrated the feasibility of offering HIV testing in community pharmacies. ${ }^{16}$ Importantly, $96 \%$ of participants accepted the idea of pharmacists performing the tests, which involves a finger-stick. This percentage was comparable to acceptance of physicians performing the test. Point-of-care testing in community pharmacies in the Netherlands also has been shown to be feasible for creatinine testing to monitor kidney function. ${ }^{17}$ This study used POCT to examine creatinine levels in a capillary blood sample from elderly patients on maintenance therapy for diabetes or cardiovascular disease with drugs excreted by the kidney. A pharmacist calculated the patient's renal function and the results were used for adjusting drug dosages. Consultation with the patient's physician was done as appropriate. Point-of-care testing in mental health settings in Minnesota, together with interprofessional partnerships and pharmacist management of medications, may improve the management of metabolic risks in patients taking antipsychotics. ${ }^{18} \mathrm{Al}-$ though there was no difference in metabolic syndrome and related conditions in the control and POCT groups, the authors concluded that POCT successfully identified a high proportion of patients with these disorders and that this information had the potential to improve long-term risks in these patients.

Overall, pharmacists are well-positioned to provide POCT to improve patient access to clinical data and enhance disease state and medication management programs. ${ }^{19}$ Such provision can occur in community and institutional pharmacy settings and should include an emphasis on preventive care. Nevertheless, challenges to achieving the potential of POCT exist that must be addressed in the coming years. 


\section{American Journal of Pharmaceutical Education 2016; 80 (8) Article 129.}

\section{POCT CHALLENGES}

Point-of-care testing ordered by a clinician or obtained by a patient through publicly available kits have their own challenges. Furthermore, how the results are used in these situations may differ. Ideally, the pharmacist should act in collaboration with the patient and other health-care professionals to optimize the use of the data obtained and, consequently, the clinical outcomes.

Physician- and pharmacist-ordered POCT involves an expanding number of tests. Clinician-managed POCT has a significant level of control in terms of validity, interpretation, how the results are used, and incorporation of the results into the patient's record. However, it is the patient-obtained POCT that raises the most concerns such as determining whether such kits are accurate and which kits can be used by individual patients, where data from unsanctioned kits would go, what happens if patients make decisions based on a faulty understanding of the significance of the results, and how to handle data obtained from monitoring devices such as smart watches. Answers to these and other questions will be key to the broader and effective use of POCT.

Probably the greatest concern with POCT is reliability. The CLIA-waived category guarantees simplicity and that problems adversely affecting patient care pose minimal risk, but not reliability. Simplicity is deceptive, however, and there are many ways a result can be wrong. Importantly, POCT results may not be comparable to those from a central laboratory. While the results should be comparable within the same test system, POCT may use different technology than that in central laboratories. Differences in the quality of the specimen, its potential storage before use, and other technical factors may also affect the results. As POCT becomes more widely used, pharmacists are positioned to investigate this critical reliability concern through collaborative research projects.

Another major challenge with patient-initiated POCT is interpreting results. Although "normal" parameters can be established for all tests, what constitutes normal can vary and be affected by age, gender, diet, medications, comorbidities, etc. Thus, while a nonspecialist (including the patient) may perform the test, who determines what action may need to be taken in response to the results is unclear. Traditionally, physicians order, review, interpret, and make clinical decisions based on test results. However, as POCT expands, physicians will not have the capacity to view all test results. An alternative may be pharmacists, the most accessible health-care professionals, who serve as first point of contact in terms of providing the test kits and doing an initial interpretation of the results leading to a care plan or patient referrals to appropriate follow-up care.
A third significant challenge is taking action on test results. When a pharmacist does POCT, the patient history and a physical assessment should take place before determining whether testing is appropriate. However, once determined appropriate, it is essential that action be taken on both normal and abnormal results. This can involve a direct patient care intervention (ie, starting, changing, or stopping drug therapy) by the pharmacist, if within their scope of practice, including advanced prescribing authority and collaborative arrangement or referral to an appropriate health care provider. One example studied for actionability is POCT results for infectious diseases. Such testing could benefit patients and represents an opportunity to expand services in community pharmacies. ${ }^{1}$ Interestingly, about $75 \%$ of adults who visit a physician for pharyngitis receive a prescription for an antibiotic even though less than $5 \%$ of such problems involve bacteria (most pharyngitis is viral). ${ }^{20}$ Thus, pharmacists treating only those cases of pharyngitis accompanied by a positive strep test would provide more appropriate therapy.

Although the results of POCT ordered by a clinician, including pharmacists, should be incorporated into the patient's record, connecting POCT devices to an electronic medical record, or uploading numerical results from tests performed at home or other noninstitutional locations, requires a compatible computer interface and must overcome security and privacy issues. Currently, no electronic record systems are designed to accept data from a range of health-care providers and certainly not from patients themselves. However, with advances in wireless communication, the way in which patients are monitored and the data managed will change dramatically. Already, numerous consumer devices exist to monitor exercise, heart rate, and other physiologic parameters. These devices are creating more personalized health care, ${ }^{4}$ something POCT will further and assist with as it tailors interventions to individual patients. Inevitably, the role patients take in their own health care will increase, and it is possible that relevant data from these sources may become part of a patient's record.

Increasing the role of patients in their own care is appropriate and should be encouraged. However, POCT done by nonprofessionals should be limited to simple and largely error-proof tests. But even these types of tests will yield useful data, and problems associated with data interpretation, appropriate action in response to the results, and communication of results to the patient's physician or other relevant health care professionals must be addressed. Critical future needs include resources to assist patients in understanding test results (a role pharmacists ideally fill) and data management systems that can handle 


\section{American Journal of Pharmaceutical Education 2016; 80 (8) Article 129.}

the POCT results. Until such systems are in place (which may be a long time), it is likely that patient-initiated POCT results will remain informational, though will hopefully stimulate patients to take more formal actions.

\section{FUTURE EDUCATIONAL NEEDS AND POSSIBILITIES}

Training on how to perform POCT is a potential challenge to their use, although by definition "waived" tests must be simple to perform. Nevertheless, a perceived barrier to the implementation of POCT services includes inadequate education of student and practicing pharmacists. Standards from CCAPP and ACPE either directly or indirectly mandate education in clinical laboratory tests. However, while all pharmacy programs teach students about laboratory test results, no information exists about the degree to which this education should include increased information about physical assessment, interpretation and use of laboratory values, and POCT. A detailed consideration of curricular needs for POCT for infectious diseases has been published. ${ }^{21}$ Further, pharmacy schools may need to consider their physical environment in the context of offering POCT training to students. Schools may need CLIA licensure plus compliance with US and state health and safety standards in order to train students on POC devices.

Resources for practicing pharmacists to learn about POCT are increasing rapidly. There are professional development ${ }^{22}$ and certificate programs. ${ }^{17,23}$ More programs are likely to be developed, and incorporating aspects of these programs into pharmacy school curricula should be considered.

Training for POCT done in an institutional setting (including schools of pharmacy) should include an increased emphasis on the details about the technology employed and the advantages and challenges associated with POCT. This would involve teaching theory and practice, storage and preparation of patient samples, appropriate quality control, and data recording and interpretation. At the same time, in jurisdictions where necessary, efforts should be made to change regulations to optimize the use of POCT in terms of patient care.

\section{CONCLUSION}

When pharmacists began to offer immunizations 25 years ago, the rapid uptake of this service by patients (in Alberta, over $50 \%$ of all flu immunizations were done in pharmacies after only four years) marked a turning point in the public's perception of the profession from one focused on the drug product to one focused on providing a broader range of health-care services and management. Point-ofcare testing has the potential to take this perception of pharmacists to an even higher level. Importantly, POCT provides an opportunity for pharmacists to become more directly engaged with patients in terms of monitoring and managing their care. However, realization of this potential will require a conscious effort of educators to both enhance the knowledge of student pharmacists and to provide professional development opportunities about POCT for those pharmacists already in practice.

\section{REFERENCES}

1. Gubbins PO, Klepser ME, Dering-Anderson AM, et al. Point-ofcare testing for infectious disease: opportunities, barriers, and considerations in community pharmacy. J Am Pharm Assoc. 2014; 54(2):163-171.

2. Seventh Annual Next Generation Summit. http://www. nextgenerationdx.com/pharmacy-diagnostics/. Accessed January 5, 2016.

3. Pecoraro V, Germagnoli L, Banfi G. Point-of-care testing: where is the evidence? A systematic survey. Clin Chem Lab Med. 2014;52 (3):313-324.

4. Servick K. Mind the phone. Science 2015;350(6266):1306-1309.

5. Rossi AF, Khan D. Point of care testing: improving pediatric outcomes. Clin Biochem. 2004;37(6):456-461.

6. Shafiee H, Wang S, Inci F, et al. Emerging technologies for pointof-care management of HIV infection. Annu Rev Med. 2015;66:387405.

7. Canadian Agency for Drugs and Technologies in Health. Guidance on the use of point-of-care testing of international normalized ratio for patients on oral anticoagulant therapy. CADTH Optimal Use Report 2014. http://www.cadth.ca/media/pdf/ OP0515_POC\%20INR_Recs_Report.pdf. Accessed January 5, 2016. 8. St John A. The evidence to support point-of-care testing. Clin Biochem Rev. 2010;31(3):111-119.

9. Howick J, Cals JWL, Jones C, et al. Current and future use of point-of-care tests in primary care: an international survey in Australia, Belgium, The Netherlands, the UK and the USA. BMJ Open 2014;4(8):e005611.

10. Tests granted waived status under CLIA. Centers for Medicare \& Medicaid Services. https://www.cms.gov/Regulations-andGuidance/Legislation/CLIA/downloads/waivetbl.pdf. Accessed January 5, 2016.

11. Rodis JL, Thomas RA. Stepwise approach to developing pointof-care testing services in the community/ambulatory pharmacy setting. J Am Pharm Assoc. 2006;46(5):594-604.

12. Centers for Disease Control and Prevention. Good laboratory Practices for Waived Testing Sites: Survey Findings from Testing Sites Holding a Certificate of Waiver Under Clinical Laboratory Improvement Amendments of 1988 and Recommendations for Promoting Quality Testing. MMWR. 2005;54(RR-13):1-25.

13. Carreyrou J. Hot startup Theranols has struggled with its bloodtest technology. The Wall Street Journal. http://www.wsj.com/ articles/theranos-has-struggled-with-blood-tests-1444881901 14. Shephard M, O'Brien C, Burgoyne A, et al. Review of the cultural safety of a national indigenous point-of-care testing program for diabetes management. Austral. J Primary Health. http://dx.doi. org/10.1071/PY15050.

15. Adams AJ. Toward permissionless innovation in health care. $J$ Am Pharm Assoc. 2015;55(4):359-362.

16. Weidle PJ, Lecher S, Botts LW, et al. HIV testing in community pharmacies and retail clinics: a model to expand access to screening for HIV infection. J Am Pharm Assoc. 2014;54(5):486-492. 


\section{American Journal of Pharmaceutical Education 2016; 80 (8) Article 129.}

17. Geerts AF, De Koning FH, De Vooght KM, Egberts AC, De Smet PA, van Solinge WW. Feasibility of point-of-care creatinine testing in community pharmacy to monitor drug therapy in ambulatory elderly patients. J Clin Pharm Therap. 2013;38(5):416-422.

18. Schneiderhan ME, Shuster SM, Davey CS. Twelve-month prospective randomized study of pharmacists utilizing point-of-care testing for metabolic syndrome and related conditions in subjects prescribed antipsychotics. Prim Care Companion CNS Disord. 2014;16(5). doi:10.4088/PCC.14m01669

19. Burley E, Klepser S, Klepser M. Opportunities for pharmacists to improve access to primary care through use of CLIA-waived tests. Mich Pharm. 2014;52(2):8-11

Appendix 1. Point-of-Care Testing

\section{Commonly used tests}

Glucose

Blood gases/electrolytes

Activated clotting time for high dose heparin monitoring

Urine dipsticks including pregnancy

Occult blood

Hemoglobin

Rapid strep

Available but variable use

Cardiac markers

Drug/toxicology

INR/PT

Heparin

D-Dimer test for thromboembolism

Magnesium

Lactate

Transcutaneous bilirubin

Lipids

Hemoglobin A1c

Microalbumin, creatinine

HIV

Influenza

H. pylori

Other bacteria

Emerging and future tests

Complete blood count

White blood cell count

Coagulation for transfusion algorithms

Platelet function

Microbiology - outbreaks, epidemics, MRSA

Endocrine testing to guide surgical therapy

Parathyroid hormone

ACTH

Gastrin

Growth hormone

Testosterone
20. Gonzales R, Steiner JF, Sande MA. Antibiotic prescribing for adults with colds, upper respiratory tract infections, and bronchitis by ambulatory care physicians. JAMA 1997;278(11):901-904.

21. Akinwale TP, Adams AJ, Dering-Anderson AM, Klepser ME. Pharmacy-based point-of-care testing for infectious diseases: considerations for the pharmacy curriculum. Curr Pharm Teach Learn. 2015;7(1):131-136.

22. https://www.pharmacists.ca/index.cfm/education-practiceresources/professional-development/lab-tests/; http://pharmacy. nova.edu/ce/programs/2015_Sept20_Lab.html

23. Community pharmacy-based point-of-care testing certificate. http://nacds.learnercommunity.com. Accessed January 5, 2016. 
American Journal of Pharmaceutical Education 2016; 80 (8) Article 129.

Sepsis markers

Stroke markers

Cancer markers

DNA testing

\section{POCT issues}

Accuracy and usefulness of tests

Standardization/comparability - quality control

Data management/oversight; interpretation (role for pharmacists)

Patient and health provider access

Where and how are data stored and distributed

Where should POCT be done

Home

Pharmacy

Emergency department

Nursing home

Hospital

Clinic

Physicians' office

Abbreviations: $\mathrm{ACTH}=$ drenocorticotropic hormone; $\mathrm{HIV}=$ human immunodeficiency virus; $\mathrm{INR}=$ international normalized ratio; MRSA = methicillin-resistant Staphylococcus aureus; $\mathrm{PT}=$ prothrombin time 